NASA Technical Memorandum 101399

ICOMP-88-20

\title{
A Near-Wall Turbulence Model and Its Application to Fully Developed Turbulent Channel and Pipe Flows
}

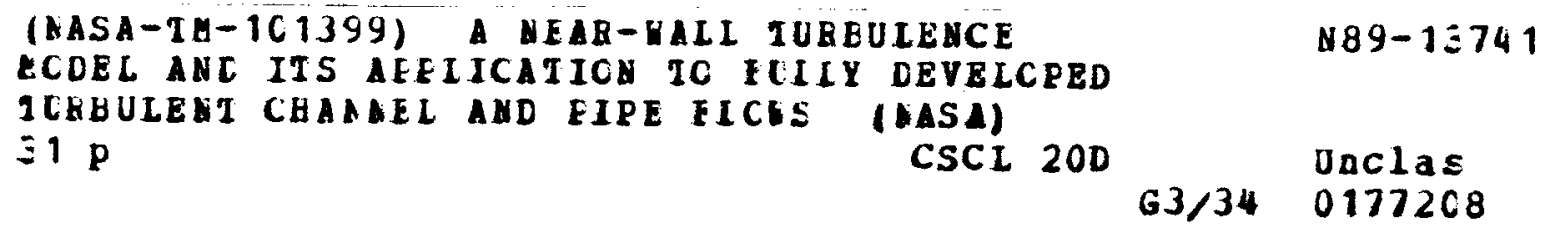

S.-W. Kim

Institute for Computational Mechanies in Propulsion

Lewis Research Center

Cleveland, Ohio

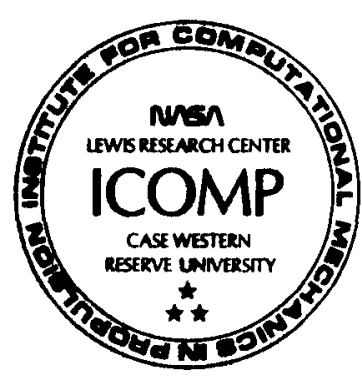


A NEAR-WALL TURBULENCE MODEL AND ITS APPLICATION TO

FULLY DEVELOPED TURBULENT CHANNEL AND PIPE FLOWS

\author{
S. -W. Kim \\ Institute for Computational Mechanics in Propulsion \\ Lewis Research Center \\ Cleveland, Ohio 44135
}

\begin{abstract}
Summary
A near-wall turbulence model and its incorporation into a multipletime-scale turbulence model are presented in this report. In the method, the conservation of mass, momentum, and the turbulent kinetic energy equations are integrated up to the wall; and the energy transfer rate and the dissipation rate inside the near-wall layer are obtained from algebraic equations. The algebraic equations for the energy transfer rate and the dissipation rate inside the near-wall layer have been obtained from a k-equation turbulence model and the near wall analysis. A fully developed turbulent channel flow and fully developed turbulent pipe flows were solved using a finite element method to test the predictive capability of the turbulence model. The computational results compared favorably with experimental data. It is also shown that the present turbulence model could resolve the over-shoot phenomena of the turbulent kinetic energy and the dissipation rate in the region very close to the wall.
\end{abstract}

*Work funded under Space Act Agreement C99066G. 


\section{Nomenclature}

$c_{p \ell}$

$c_{t \ell}$

$c_{\mu \mathrm{f}}$

$\mathrm{c}_{\mu}$

$\mathrm{f}_{\mu}$

$\mathrm{f}_{\epsilon}$

k

$k_{p}$

$\mathrm{k}_{\mathrm{t}}$

$\mathrm{k}^{+}$

$\operatorname{Pr}$

$R_{t}$

r

u

$\mathrm{u}^{+}$

$\mathrm{u}_{\tau}$

$\mathrm{u}^{2}$

$\overline{u^{\prime} v^{\prime}}$

$\overline{u^{\prime} v^{\prime}}+$

$\mathrm{v}$

$v_{s}$

y

$y^{+}$

$\epsilon$

$\epsilon_{\mathrm{p}}$

Turbulence model constants for $\epsilon_{\mathrm{p}}$ equation, $(\ell=1,3)$

Turbulence model constants for $\epsilon_{t}$ equation, $(\ell=1,3)$

A constant coefficient $(=0.09)$

A coefficient used in eddy viscosity equation

Wall damping function for eddy viscosity equation

Wall damping function for dissipation rate

Total turbulent kinetic energy, $\left(k=k_{p}+k_{t}\right)$

Turbulent kinetic energy of eddies in the production range

Turbulent kinetic energy of eddies in the dissipation range

Normalized turbulent kinetic energy $\left(-k / u_{\tau}{ }^{2}\right)$

Production rate of turbulent kinetic energy

Turbulent Reynolds number $\left(=\mathrm{k}^{2} /(\nu \epsilon)\right)$

Radial distance

Time averaged velocity

Non-dimensional velocity $\left(=u / u_{\tau}\right)$

Friction velocity $\left(-\sqrt{ }\left(\tau_{\mathrm{w}} / \rho\right)\right)$

Normal Reynolds stress, R.M.S. value of the fluctuating axial velocity

Reynolds stress

Normalized Reynolds stress $\left(=\overline{u^{\prime} v^{\prime}} / u_{\tau}{ }^{2}\right)$

Time averaged velocity in the $y$-coordinate direction

turbulence velocity scale

normal distance from the wall

Wall coordinate $\left(=u_{\tau} y / v\right)$

Dissipation rate

Transfer rate of turbulent kinetic energy from the production range to the dissipation range 


$\begin{array}{ll}\epsilon_{t} & \text { Dissipation rate of turbulent kinetic energy } \\ \epsilon_{\mathrm{w}} & \text { Dissipation rate inside the near-wall layer } \\ \epsilon^{+} & \text {Non-dimensional dissipation rate }\left(-\nu \epsilon / u_{\tau}{ }^{4}\right) \\ \kappa & \text { von Karman constant } \\ \ell & \text { turbulence length scale } \\ \ell_{\mathrm{m}} & \text { mixing length } \\ \nu & \text { kinematic viscosity of fluid } \\ \nu_{t} & \text { turbulent eddy viscosity } \\ \sigma_{\mathrm{kp}} & \text { Turbulent Prandtl number for } k_{\mathrm{p}} \text { equation } \\ \sigma_{\mathrm{kt}} & \text { Turbulent Prandtl number for } k_{\mathrm{t}} \text { equations } \\ \sigma_{\epsilon \mathrm{p}} & \text { Turbulent Prandtl number for } \epsilon_{\mathrm{p}} \text { equation } \\ \sigma_{\epsilon t} & \text { Turbulent Prandtl number for } \epsilon_{\mathrm{t}} \text { equation } \\ \tau_{\mathrm{w}} & \text { Wall shearing stress }\end{array}$




\section{Introduction}

For wall bounded turbulent flows in the equilibrium state, the logarithmic velocity profile prevails in the near-wall region 1 . The most widely used wall function methods in numerical calculations of turbulent flows have been derived from the logarithmic velocity profile based on the experimental observation that the turbulence in the near-wall region can be described in terms of the wall shearing stress. The wall function methods are not valid at or near the separation point since the turbulence in this region can not be described in terms of the wall shearing stress. For unsteady turbulent flows, the logarithmic velocity profile no longer prevails in the near-wall or in the wake region ${ }^{2}$, therefore the wall function methods can not be applied. Other occasions in which the wall function methods become invalid include low turbulent Reynolds number flows in which the effect of molecular viscosity become important and flows over surfaces with mass injection and/or suction. The objective of this study is to eatablishes a near-wall turbulence model which can be used in place of

wall function methods. Further application of the present near-wall

turbulence model for complex turbulent flows such as the turbulent flow over a curved hill ${ }^{3}$ and the turbulent boundary layer - shock wave interactions ${ }^{4-5}$ can be found in Reference 6 .

Numerous efforts have been made to include the near-wall low turbulent Reynolds number region in turbulent flow computations. Various turbulence models which include the near-wall low turbulence region can be classified as two- or multi-layer turbulence models ${ }^{7-10}$, low Reynolds number turbulence models, and partially low Reynolds number turbulence models. The advantages and disadvantages of these various methods are discussed in the following. 
In the two- or multi-layer turbulence models, the turbulence in the near-wall layer has been described by algebraic expressions ${ }^{7-10}$. In these turbulence models, the turbulent kinetic energy and the dissipation rate in the near-wall layer have been constructed by piecewise continuous functions. As a consequence, the computational results may depend on the location of the partition between the near-wall layer and the fully turbulent region ${ }^{8}$. In some of these methods, a quadratic variation of the turbulent kinetic energy has been assumed in the near-wall region and the dissipation rate has been derived from the logarithmic velocity profile equation ${ }^{4}$. This class of methods is advantageous in studying the near-wall turbulence structure for equilibrium turbulent flows. However, these methods become invalid if the logarithmic velocity profile does not prevail in the near wall region. The underlying assumption that the turbulent kinetic energy is proportional to square of the distance from the wall inside the near-wall layer is also questionable, since the quadratic variation of the turbulent kinetic energy is valid only inside the viscous sublayer and become invalid as the fully turbulent region is approached.

In the low Reynolds number turbulence models, the high Reynolds number turbulence models have been extended to include the near-wall low turbulence effect ${ }^{11}$. In this class of methods, the near-wall low turbulence effects have been incorporated into the high Reynolds number turbulence models by using the wall damping functions. These wall damping functions have been derived mostly from numerical experiments in such a way that the low Reynolds number turbulence models could reproduce the experimentally observed turbulent flow phenomena in the near-wall region. In this class of methods, a significant number of grid points has to be assigned in the near wall region in order to resolve the stiff dissipation rate equation. 
Aside from the above two classes of methods, a new approach has been used in Chen and Patel ${ }^{12}$ to solve complex turbulent flow. In the method, only the turbulent kinetic energy equation of the $k-\epsilon$ turbulence model has been extended to include the near-wall low turbulence effect and the dissipation rate inside the near-wall layer has been prescribed algebraically. The dissipation rate equation has been obtained from a k-equation turbulence model ${ }^{13}$. Thus the turbulent kinetic energy and the dissipation rate varies smoothly from the wall toward the outside fully turbulent region. In this case, it would be more appropriate to classify the turbulence model as a partially low Reynolds number turbulence model to distinguish it from the other two classes of methods.

It has been shown previously that the high Reynolds number multiple-time-scale turbulence model ${ }^{16}$ yielded significantly improved computational results for a class of complex turbulent flows such as a wall jet flow 17 , a wake-boundary layer interaction flow ${ }^{18}$, a confined coaxial jet without swirl ${ }^{19}$, and a confined coaxial swirling jet ${ }^{20}$, to name a few. In the single-time-scale turbulence models such as the $k-\epsilon$ turbulence models and the Reynolds stress turbulence models, a single-time-scale is used to express both the turbulent transport and the dissipation of the turbulent kinetic energy. However, this practice is inconsistent with physically observed turbulence in a sense that the turbulent transport is related to the time scale of energy containing large eddies and the dissipation rate of turbulent kinetic energy is related to the time scale of fine scale eddies in the dissipation range. The single time-scale turbulence models have been used most widely and yielded quite accurate computational results for many turbulent flows; however, the predictive capability degenerated significantly for complex turbulent flows with 
strong inequilibrium turbulence. In the multiple-time-scale turbulence models 16,21-24, the turbulent transport of mass and momentum has been described using the time scale of the large eddies; and the dissipation rate has been described using the time scale of the fine scale eddies. The improved computational results obtained by using the multiple-time-scale turbulence model for complex turbulent flows can be attributed to the above discussed physically consistent nature of the turbulence models.

It has been shown that the $\mathrm{k}$-equation turbulence models yielded highly accurate computational results for a class of standard turbulent boundary layer flows 25 , turbulent flows with drag reduction ${ }^{26}$, an unsteady fully developed turbulent channel flow 27 , and fully developed unsteady turbulent pipe flows ${ }^{2}$. However, the k-equation turbulence model itself is less useful for separated and/or swirling turbulent flows with complex geometry due to lack of a systematic method to evaluate the turbulence length scale.

The near-wall turbulence model presented in this report belongs to the partially low Reynolds number turbulence models. In the model, the turbulence structure in the near-wall low turbulence region has been solved by extending the turbulent kinetic energy equations to include the low turbulence effect and the energy transfer rate and the dissipation rate have been prescribed algebraically. The energy transfer rate and the dissipation rate equations in the near-wall layer have been obtained from the k-equation turbulence model ${ }^{13}$. The rest of the flow domain has been solved by the high Reynolds number multiple-time-scale turbulence model 16 . Further advantages of this approach originating from physical and numerical considerations are discussed in the following section.

Example problems considered include: a fully developed turbulent channel flow ${ }^{14}$ at Reynolds number (Re) of 30,800 and turbulent pipe flows ${ }^{15}$ 
for $\operatorname{Re}=50,000$ and 500,000 . The computational results compared favorably with experimental data globally. More importantly, significantly improved computational results for the turbulence structure in the near-wall region, including the experimentally observed over-shoot phenomena of the turbulent kinetic energy and the dissipation rate, have been obtained.

\section{NEAR-WALL TURBULENCE MODEL}

The turbulence in external flows and that in near-wall boundary layer flows have quite different length scales 28,29 . The turbulence length scale of the external flows is related to the flow field characteristics. On the other hand, the turbulence length scale of boundary layer flows is strongly related to the normal distance from the wall. This nature of the wall bounded turbulent flows can be described quite naturally by the partially low Reynolds number turbulence models. The same purpose could be achieved by the low Reynolds number turbulence models as more experimental data become available. However, the gradient of the dissipation rate is the most stiff in the near-wall region, and a great number of grid points has to be used in the region for the low Reynolds number turbulence models to resolve the dissipation rate. Therefore, the partially low Reynolds number turbulence models seem to be more advantageous as compared with the low Reynolds number turbulence models, unless the low Reynolds number turbulence models can describe the wall bounded turbulent flows more accurately.

\section{Eddy Viscosity Equation in the Near-Wall Region}

In the Prandtl-Kolmogorov theory, the eddy viscosity is given as;

$$
\nu_{t}=v_{s} \ell
$$


where $v_{s}$ and $\ell$ are the turbulence velocity and length scales, respectively. The velocity scale has been most frequently represented by the square root of the turbulent kinetic energy. The turbulent length scale in the fully turbulent region of wall bounded turbulent flows is given as ${ }^{11,25}$;

$$
\begin{aligned}
& \ell=c_{\mu f^{1 / 4} \ell_{\mathrm{m}}} \\
& \ell_{\mathrm{m}}=\kappa \mathrm{y}
\end{aligned}
$$

where $c_{\mu f}(-0.09)$ is a constant, $\ell_{m}$ is the mixing length, $\kappa$ is the von Karman constant, and $y$ is the normal distance from the wall. The fully turbulent region extends from $y^{+} \approx 30$ up to $y^{+} \approx-300$, where $y^{+}=u^{\tau} y / \nu$ is the wall coordinate, $u_{\tau}=\sqrt{ }\left(\tau_{w} / \rho\right)$ is the friction velocity, $\tau_{w}$ is the wall shearing stress, and $\nu$ is the kinematic viscosity of fluid. Thus the turbulent eddy viscosity in this fully turbulent region is given as;

$$
\nu_{t}=\mathrm{k}^{1 / 2} \ell
$$

where $k$ is the turbulent kinetic energy.

The instantaneous velocity in the viscous sublayer region can be written as ${ }^{11}$;

$$
\left.\begin{array}{c}
u^{\prime}=a_{1} y+a_{2} y^{2}+\ldots . . \\
v^{\prime}=\quad b_{2} y^{2}+. . . \cdot \\
w^{\prime}=c_{1} y+c_{2} y^{2}+\ldots . . .
\end{array}\right\}
$$

where the coefficients $a_{i}, b_{i}$, and $c_{i}$ are functions of time such that $a_{i}=$ 
$b_{i}=c_{i}=0$, and the over-bar denotes time average. Note that the fluctuating normal velocity component grows in proportion to the square of the distance from the wall due to the wall proximity effect. The turbulence in the very closed to the wall region, i.e., $y \approx 0$ can be written as;

$$
\begin{aligned}
& k=\frac{1}{2}\left(\overline{u^{\prime 2}}+\overline{v^{\prime 2}}+\overline{w^{\prime 2}}\right)=\frac{1}{2}\left(\overline{a_{1}^{2}}+\overline{c_{1}^{2}}\right) y^{2}+\ldots . .
\end{aligned}
$$

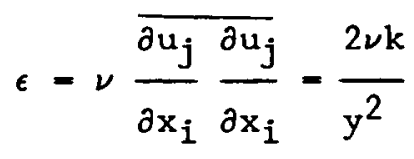

$$
\begin{aligned}
& \overline{u^{\prime} v^{\prime}}=\overline{a_{1} b_{2}} y^{3}
\end{aligned}
$$

where $\epsilon$ is the dissipation rate, and $\overline{u^{\prime} v^{\prime}}$ is a component of the Reynolds stress. Due to the difficulty in measuring the turbulence quantity in the very close to the wall region, there exist only a limited number of experimental data to support the above analysis. For example, the experimental data compiled by $\mathrm{Cole}^{30}$ showed that the turbulent kinetic energy grows in proportion to the square of the distance from the wall. In the Boussinesq eddy viscosity assumption, the Reynolds stress is given as;

$$
-\overline{u_{i}{ }^{\prime} u_{j}{ }^{\prime}}=\nu_{t}\left(\frac{\partial u_{i}}{\partial x_{j}}+\frac{\partial u_{j}}{\partial x_{i}}\right)
$$

Very close to the wall, the velocity gradient is a constant. Thus eqs. and (9) indicate that the eddy viscosity is in proportion to the third power of the distance from the wall; whereas, eqs. (2-4) and (6) indicate 
that the eddy viscosity is proportional to the square of the distance from the wall in the viscous sublayer region. This difference is attributed to the fact that the molecular viscosity dominates in the viscous sublayer region and that the eddy viscosity dominates in the fully turbulent region. The turbulent viscosity equation can be modified to include the effect of molecular viscosity in the viscous sublayer region as follows ${ }^{8-12}$;

$$
\nu_{t}=f_{\mu} k^{1 / 2} \ell
$$

Thus the damping function $f_{\mu}$ has to be a linear function of the distance from the wall and has to approach unity as the fully turbulent region is approached.

Note that the eddy viscosity in the Prandtl mixing length theory is proportional to the fourth power of the distance from the wall, i.e.;

$$
\nu_{t}=\ell_{m}^{2}\left|\frac{\partial u}{\partial y}\right|
$$

where $\ell_{m}-\kappa y\left(1-\exp \left(y^{+} / A^{+}\right)\right)$and $A^{+}$is the wall damping factor. There also exists a few low Reynolds number turbulence models in which the eddy viscosity varies in proportion to the fourth power of the distance from the wall, see Reference 11 for more discussion.

In the fully turbulent region of wall bounded turbulent flows, the dissipation rate is related to the mixing length or the turbulence length scale as;

$$
\epsilon=c_{\mu f} 3 / 4 \frac{\mathrm{k}^{3 / 2}}{l_{\mathrm{m}}}
$$




$$
=c_{\mu f} \frac{k^{3 / 2}}{\ell}
$$

The dissipation rate given as eq. (12) vanishes in the region very close to the wall. However, the experimentally observed dissipation rate approaches a constant value in the region, see eq. (7). Two slightly different approaches have been used to approximate the dissipation rate in the near-wall region. In the k-equation turbulence model of Gibson et. al. 25, Hassid and Poreh ${ }^{26}$, and Acharya and Reynolds 27 , the dissipation rate in the near-wall layer has been obtained by linear combination of eqs. (7) and (12), and is given as;

$$
\epsilon_{\mathrm{w}}=c_{\mu \mathrm{f}^{3 / 4}} \mathrm{f}_{\epsilon \mathrm{h}} \frac{\mathrm{k}^{3 / 2}}{\kappa \mathrm{y}}+\frac{2 \nu \mathrm{k}}{\mathrm{y}^{2}}
$$

where $f_{\epsilon h}$ is a wall damping function varying from null on the wall to unit in the fully turbulent region. On the other hand, in the k-equation turbulence model of Wolfshtein ${ }^{13}$, the dissipation rate in the near-wall layer was given as;

$$
\epsilon_{\mathrm{w}}=\frac{1}{\mathrm{f}_{\epsilon}} \frac{c_{\mu \mathrm{f}^{3 / 4} \mathrm{k}^{3 / 2}}}{\kappa \mathrm{y}}
$$

where $f_{\epsilon}$ has been chosen so that the dissipation rate would approach to the value given in eq. (7) for $y \approx 0$ and $f_{\epsilon}$ take unit value in the fully turbulent region. The fully turbulent region with equilibrium turbulence extends from $y^{+} \approx 30$ to $y^{+} \approx 300$ for wall bounded flows. Eq. (14) has also been used in the partially low Reynolds number $\mathrm{k}-\epsilon$ turbulence model of Chen and 
Patel ${ }^{12}$ for numerical computations of separated turbulent flows. Eq. (14) has been used in the present study, and partial justification is presented in the following paragraph.

Consider the turbulent kinetic energy equation given as;

$$
\left.u_{j} \frac{\partial k}{\partial x_{j}}-\frac{\partial}{\partial x_{j}}\left(\nu+\frac{\nu_{t}}{\sigma_{k}}\right) \frac{\partial k}{\partial x_{j}}\right)=\operatorname{Pr}-\epsilon
$$

where $\sigma_{k}$ is the turbulent Prandtl number for the $k$-equation and $\operatorname{Pr}=-\overline{u_{i}{ }^{\prime} u_{j}{ }^{\prime}}\left(\partial u_{i} / \partial x_{j}\right)$ is the production rate. Very close to the wall $(y \approx 0)$, the molecular viscosity dominates over the turbulent eddy viscosity, and the convection term is found to be negligible compared with the diffusion term from an order of magnitude analysis ${ }^{1}$. Then the analytical solution of eq. (15) for $y \approx 0$ is given as eq. (7). For $y>0$, the turbulent eddy viscosity grows in proportion to the third power of the distance from the wall. Hence it can be argued that the dissipation rate given as eq. (7), which is the analytical solution of eq. (15) for $y \approx 0$, may valid only at very close to the wall. However, the dissipation rate given as eq. (7) retains significant magnitude even in the fully turbulent region, i.e. $y^{+} \approx 100$. For this reason, the work of Wolfshtein ${ }^{13}$ and Chen and Patel ${ }^{12}$ has been followed herein. In the present study, the near-wall damping function for eq. (14) is given as;

$$
f_{\epsilon}=1-\operatorname{Exp}\left(-A_{\epsilon} R_{t}\right)
$$

where $R_{t}=k^{2} /(\nu \epsilon)$ is the turbulent Reynolds number and $A_{\epsilon}=c_{\mu} f^{3 / 2} /\left(2 \kappa^{2}\right)$. For $y \approx 0$, eq. (14) takes the limit value given as eq. (7). 
Substituting eq. (12) into eq. (10) yields:

$$
\nu_{t}=c_{\mu f} f_{\mu} \frac{k^{2}}{\epsilon}
$$

and substituting $\epsilon_{\mathrm{w}}$, eq. (14), for $\epsilon$ in eq. (17) yields the eddy viscosity equation for the near-wall layer given as;

$$
\nu_{t}=c_{\mu f} f_{\mu} \frac{k^{2}}{\epsilon_{w}}
$$

where $f_{\mu}=1-\exp \left(A_{1} \sqrt{ } R_{t}+A_{2} R_{t}{ }^{2}\right)$ is a linear function of the distance from the wall in the viscous sublayer region and become unity in the fully turbulent region. $A_{1}=0.025$ and $A_{2}=0.00001$ have been used for the near wall layer in the present study.

For near-wall equilibrium turbulent flows, the production rate is approximately equal to dissipation rate $\left(\epsilon_{t}\right)$, and hence the energy transfer rate $\left(\epsilon_{p}\right)$ from the low wave number production range to the high wave number dissipation range has to be approximately equal to both of them. The energy transfer rate and the dissipation rate inside the near-wall layer are given as ;

$$
\epsilon_{p}=\epsilon_{t}=\epsilon_{w}=\frac{1}{f_{\epsilon}} \frac{c_{\mu f^{3 / 4} k^{3 / 2}}}{\kappa y}
$$

Note that the production rate vanishes on the wall and grows to full strength at $y^{+} \approx 15$. Hence eq. (19) may not be a good approximation for $0<y^{+}<15$. However, use of the vanishing boundary condition for the 
turbulent kinetic energy on the wall yields the growth rate of the turbulent kinetic energy and the production rate which is in good agreement with experimental data as well as theoretical analysis.

\section{Multiple-Time-Scale Turbulence Equations}

For clarity, the partially low Reynolds number multiple-time-scale turbulence equations are summarized below. The partition between the near-wall region and the fully turbulent outer region can be located between $\mathrm{y}^{+}$greater than 40 and less than 300 approximately. The turbulent kinetic energy equations for the entire flow domain are given as;

$$
\begin{aligned}
& u_{j} \frac{\partial k_{p}}{\partial x_{j}}-\frac{\partial}{\partial x_{j}}\left(\left(\nu+\frac{\nu_{t}}{\sigma_{k p}}\right) \frac{\partial k_{p}}{\partial x_{j}}\right)=\operatorname{Pr}-\epsilon_{p} \\
& u_{j} \frac{\partial k_{t}}{\partial x_{j}}-\frac{\partial}{\partial x_{j}}\left(\left(\nu+\frac{\nu_{t}}{\sigma_{k t}}\right) \frac{\partial k_{t}}{\partial x_{j}}\right)=\epsilon_{p}-\epsilon_{t}
\end{aligned}
$$

where $u_{j}$ is the time averaged velocity in the $j$-th spatial coordinate direction, $\nu$ is the kinematic viscosity of the fluid, $\nu_{t}$ is the turbulent eddy viscosity, and $\sigma_{\mathrm{kp}}-0.75$ and $\sigma_{\mathrm{kt}}-0.75$ are the turbulent Prandt numbers for the $k_{p}$ and $k_{t}$ equations, respectively.

The energy transfer rate and the dissipation rate in the near-wall layer are given as eq. (19). The convection-diffusion equations for the energy transfer rate and the dissipation rate in the rest of the flow domain are given as; 


$$
\begin{aligned}
& u_{j} \frac{\partial \epsilon_{p}}{\partial x_{j}}-\frac{\partial}{\partial x_{j}}\left(\left(\nu+\frac{\nu_{t}}{\sigma_{\epsilon}}\right) \frac{\partial \epsilon_{p}}{\partial x_{j}}\right)=c_{p 1} \frac{\operatorname{Pr}^{2}}{k_{p}}+c_{p 2} \frac{\operatorname{Pr} \epsilon_{p}}{k_{p}}-c_{p} \frac{\epsilon_{p}}{k_{p}} \\
& \left.u_{j} \frac{\partial \epsilon_{t}}{\partial x_{j}}-\frac{\partial}{\partial x_{j}}\left(\nu+\frac{\nu_{t}}{\sigma_{\epsilon t}}\right) \frac{\partial \epsilon_{t}}{\partial y}\right)=c_{t 1} \frac{\epsilon^{2}}{k_{t}}+c_{t 2} \frac{\epsilon_{p} \epsilon_{t}}{k_{t}}-c_{t 3} \frac{\epsilon_{t}{ }^{2}}{k_{t}}
\end{aligned}
$$

where $\sigma_{\epsilon \mathrm{p}}=1.15$ and $\sigma_{\epsilon t}=1.15$ are the turbulent Prandtl numbers for the $\epsilon_{\mathrm{p}}$ and $\epsilon_{t}$ equations, respectively, and $c_{p l}(\ell=1,3)$ and $c_{t \ell}(\ell=1,3)$ are turbulence model constants. These model constants are given as; $c_{p 1}=0.21$, $c_{p 2}=1.24, c_{p 3}=1.84, c_{t 1}=0.29, c_{t 2}=1.28$, and $c_{t 3}=1.66$. Detailed derivation of these model constants can be found in Reference 16 .

The eddy viscosity equation in the near-wall region is given as eq. (13); and the eddy viscosity in the rest of the flow domain is given as;

$$
\nu_{\mathrm{t}}=\mathrm{c}_{\mu \mathrm{f}} \frac{\mathrm{k}^{2}}{{ }_{\epsilon \mathrm{p}}}
$$

\section{COMPUTATIONAL RESULTS}

The turbulent boundary layer flows were solved by a finite element method $^{31}$. It has been shown in Reference 31 that the finite element method could solve a wide range of laminar boundary layer flows, such as the Blasius flat plate flow, the retarded Howarth flow, flow over a wedge, plane stagnation flow, flow over a circular cylinder, flow in the wake of a flat plate, uniform suction flow over a flat plate, flow over a cone, and flow over a sphere, as accurately as any available numerical methods including the semi-analytical methods (i.e., a fourth-order Runge-Kutta method $^{1}$ ). Convergence study of the finite element method can be found in reference 31 , and implementaion of the method for turbulent flows can be 
found in Reference 32. For completeness, the computational procedure relevant to the present study is described briefly below.

In each iteration, the momentum equation and the turbulence equations were solved sequentially with under-relaxation. The systems of equations have been solved iteratively until the maximum relative error of each turbulent flow variable became less than the specified convergence criterion for each flow variable. Each system of equations was solved by a penta-diagonal matrix algorithm (PDMA). Since the discrete system of equations contained five diagonal entries, the PDMA yielded exact solution for each system of equations. The convergence criterion used is given as;

$$
\left|\left(a_{i, j}^{n+1}-a_{i, j}^{n}\right) / A_{i}^{n+1}\right|<e_{i}, \quad j=1, N,
$$

where $i\left(-u, k_{p}, \epsilon_{p}, k_{t}\right.$, or $\left.\epsilon_{t}\right)$ denotes each flow variable; $A_{i}$ denotes the maximum magnitude of the $i-t h$ flow variable; $N$ denotes the number of degrees of freedom for each flow variable; $e=1 \times 10^{-7}$ has been used for velocity; and $e=5 \times 10^{-5}$ has been used for the rest of flow variables.

\section{Fully Developed Channe1 Flow}

The experimental data for the fully developed channel flow can be found in Laufer ${ }^{14}$. The Reynolds number based on the center line mean velocity $(7.07 \mathrm{~m} / \mathrm{sec})$ and the half of the channel width (0.0635 meters) was approximately 30,800 . The partition between the near-wall layer and the fully turbulent region has been located at $y^{+} \approx 100$. The near-wall layer has been discretized by 20 grid points; and the rest of the flow domain, by 40 grid points. The Dirichlet boundary condition $\left(u-k_{p}-k_{t}-0\right)$ has been prescribed at the wall; and the vanishing gradient boundary condition, at 
the center line of the channel. The pressure gradient estimated from the pressure measurement and the wall shearing stress measurement were -1.41 $\mathrm{kg}-\mathrm{m} / \mathrm{sec}^{2}-\mathrm{m}^{3}$ and $-1.40 \mathrm{~kg}-\mathrm{m} / \mathrm{sec}^{2}-\mathrm{m}^{3}$, respectively. In the present computation, $\mathrm{dp} / \mathrm{dx}=-1.405 \mathrm{~kg}-\mathrm{m} / \mathrm{sec}^{2}-\mathrm{m}^{3}$ has been used. The converged solution has been obtained after approximately 400 iterations.

The computational results for the velocity, the turbulent kinetic energy, and the Reynolds stress are compared with the experimental data in Figure 1. The velocity profile compared less favorably with the experimental data. The under-predicted velocity profile may due to the inaccurate pressure gradient. The magnitude and location of the maximum turbulent kinetic energy compared favorably with experimental data. It can be seen from the Reynolds stress profile that the present turbulence model yielded correct distribution of the turbulent viscosity.

The normalized velocity, the turbulent kinetic energy, and the Reynolds stress inside the near-wall layer are shown in Figure 2; and the normalized dissipation rate, the ratio of $\operatorname{Pr} / \epsilon_{t}$, and the wall damping function are shown in Figure 3. The calculated magnitude and the location of the overshoots of the turbulent kinetic energy and the dissipation rate were in good agreement with the experimental data. The rest of computational results such as the ratio of production rate to dissipation rate of the turbulent kinetic energy and the wall damping function compared favorably with the semi-emperical data. ${ }^{11}$ It is shown in Reference 11 that only a few low Reynolds number turbulence models could yield the turbulent kinetic energy profile with overshoot in the near-wall region. It can be found in Reference 32 that the global computational results obtained by using various turbulence models with wall function methods compared satisfactorily with experimental data. However, the correct magnitude of 
the overshoot of the turbulent kinetic energy and the dissipation rate have seldom been obtained.

\section{Fully Developed Pipe Flows}

The fully developed pipe flows at Reynolds numbers of 50,000 and 500,000 are considered below. The experimental data can be found in Laufer ${ }^{14}$. The Reynolds number was based on the diameter of pipe $(0.24688$ meters) and the center line mean velocity. The center line velocities were $3.048 \mathrm{~m} / \mathrm{sec}$ and $30.48 \mathrm{~m} / \mathrm{sec}$ for $\operatorname{Re}=50,000$ and 500,000 , respectively. In each case, the partition between the near-wall layer and the fully turbulent region has been located at $y^{+} \approx 100$. For $\operatorname{Re}=50,000$, the near-wall layer has been discretized by 20 grid points; and the rest of the flow domain, by 40 grid points. For $\operatorname{Re}=500,000$, the near-wall layer has been discretized by 20 grid points; and the rest of the flow domain, by 60 grid points. For both cases, the Dirichlet boundary condition ( $u-k_{p}=k_{t}=0$ ) has been prescribed at the wall; and the vanishing gradient boundary condition, at the center line of the pipe. The pressure gradients estimated from the pressure measurements were $-0.32 \mathrm{~kg}-\mathrm{m} / \mathrm{sec}^{2}-\mathrm{m}^{3}$ and -23.05 $\mathrm{kg}-\mathrm{m} / \mathrm{sec}^{2}-\mathrm{m}^{3}$ for $\mathrm{Re}=50,000$ and 500,000 , respectively. The converged solutions have been obtained after approximately 450 iterations for both cases.

The computational results for the velocity, the turbulent kinetic energy, and the Reynolds stress for $\operatorname{Re}-50,000$ are compared with experimental data in Figure 4. For this case, the computational results compared favorably with the experimental data. The Reynolds stress profile was slightly under-predicted, however, the difference was negligible. The normalized velocity, the turbulent kinetic energy, and the Reynolds stress 
inside the near-wall layer are shown in Figure 5; and the normalized dissipation rate, the ratio of $\operatorname{Pr} / \epsilon_{t}$, and the wall damping function are shown in Figure 6 . The calculated magnitude and the location of the overshoots of the turbulent kinetic energy and the dissipation rate were in excellent agreement with the experimental data.

The computational results for the velocity, the turbulent kinetic energy, and the Reynolds stress for $\operatorname{Re}=500,000$ are compared with experimental data in Figure 7. It was found that the mean velocity profile was severely under-predicted. Again, this may due to the inaccurate pressure gradient as in the fully developed channel flow case. The thickness of the near-wall layer (i.e., $y^{+}<100$ ) for $\operatorname{Re}=500,000$ case was approximately one order of magnitude smaller than that of $\operatorname{Re}=50,000$ case. Both the calculated and the measured turbulent kinetic energy profiles exhibited strong peak in the region very close to the wall.

The normalized velocity, turbulent kinetic energy, and Reynolds stress inside the near-wall layer are shown in Figure 8; and the normalized dissipation rate, ratio of $\operatorname{Pr} / \epsilon_{t}$, and wall damping function are shown in Figure 10. Again, the calculated magnitude and the location of the overshoots of the turbulent kinetic energy and the dissipation rate were in excellent agreement with the experimental data.

\section{Conclusions and Discussion}

A near-wall turbulence mode1 and numerical computations of fully developed turbulent channel flows and pipe flows using a partially low Reynolds number multiple-time-scale turbulence model have been presented.

It has been shown that the present turbulence model yielded accurate computational results for the example flows considered. The correct 
magnitude and location of the overshoots of the turbulent kinetic energy and the dissipation rate have been obtained only by a few low Reynolds number turbulence models ${ }^{11}$; and have seldom been obtained by the two- or multi-layer turbulence models ${ }^{33}$. The turbulent kinetic energy and the dissipation rate overshoots obtained by using the present turbulence model were in excellent agreement with experimental data. The rest of the computational results in the near-wall region such as the normalized velocity profile, the Reynolds stress profile, the ratio of production rate to dissipation rate, and the wall damping function compared favorably with experimental data.

In the two- or multi-layer turbulence models, the turbulent kinetic energy in the near-wa11 layer is constructed by piecewise continuous functions; and the dissipation rate, by discontinuous functions. In the partially low Reynolds number turbulence models and in the low Reynolds number turbulence models, the turbulent kinetic energy and the dissipation rate vary smoothly from the wall toward the outside fully turbulent region. In this regard, the partially low Reynolds number turbulence models and the low Reynolds number turbulence models are more consistent with the experimentally observed distribution of the turbulent kinetic energy and the dissipation rate than the two- or multi-layer turbulence models are. A significant number of grid points has to be assigned in the near-wall layer for numerical computation of turbulent flows with the low Reynolds number turbulence models. However, with the use of partially low Reynolds number turbulence models, less number of grid points need to be assigned inside the near-wall layer and the stiff dissipation rate in the region need not be solved numerically. Therefore, many numerical codes can be made more robust and more efficient with the use of the partially low Reynolds number 
turbulence models.

\section{$\underline{\text { References }}$}

1. White, F. M., Viscous Fluid Flow, McGraw-Hill, New York, 1974.

2. Tu, S. W., and Ramaprian, B. R., "Fully Developed Periodic Turbulent Pipe Flow. Part I, Main Experimental Results and Comparison with Predictions," J. Fluid Mechanics, Vo1. 137, pp. 31-58, 1983.

3. Baskaran, V., Smits, A. J., and Joubert, P. N., "A Turbulent Flow over a Curved Hill, Part I. Growth of an Internal Boundary Layer, " J. Fluid Mech., Vo1. 182, pp. 47-83, 1987.

4. Bachalo, W. D., and Johnson, D. A., "An Investigation of Transonic Turbulent Boundary Layer Separation Generated on an Axisymmetric Flow Mode1," AIAA Paper 79-1479, 1979.

5. Horstman, C. C., Settles, G. S., Bogdonoff, S. M., and Hung, C. M., "Reynolds Number Effects on Shock-Wave Turbulent Boundary-Layer Interactions," J.AIAA, Vol. 15, No. 8, pp. 1152-1158, 1977.

6. Kim, S.-W., "Application of a Near-Wall Turbulence Mode1 to Shock Wave - Turbulent Boundary Layer Interaction Flows," In preparation, 1988 .

7. Amano, R. S., "Development of a Turbulent Near-Wall Model and Its Application to Separated and Reattached Flows," Numerical Heat Transfer, Vol. 7, pp. 59-75, 1984.

8. Gorski, J. J., "A New Near-Wall Formulation for the k- $\epsilon$ Equations of Turbulence," AIAA-86-0556, AIAA 24th Aerospace Sciences Meeting, Reno, Nevada, 1986.

9. Kim, S.-W., and Chen, C.-P., "A Finite Element Computation of Complex Turbulent Boundary Layer Flows with a Two-Layer Multiple-Time-Scale Turbulence Mode1," NASA CR, In print, 1988.

10. Viegas, J. R., Rubesin, M. W., and Horstman, C. C., "On the Use of Wall Functions as Boundary Conditions for Two-Dimensional Separated Compressible Flows," AIAA-85-0180, AIAA 23rd Aerospace Sciences Meeting, Reno, Nevada, 1985.

11. Patel, V. C., Rodi, W., and Scheuerer, G., "Turbulence Models for Near Wa11 and Low Reynolds Number Flows: A Review", J. AIAA, 23, pp. $1308-1319$ (1985).

12. Chen, H. C., Patel, V. C., "Practical Near-Wall Turbulence Models for Complex Flows Including Separation," AIAA-87-1300, 1987.

13. Wolfshtein, M., "The Velocity and Temperature Distribution in One-Dimensional Flow with Turbulence Augmentation and Pressure 
Gradient," Int. J. Heat and Mass Transfer, Vo1 12, pp. 301-318, 1969.

14. Laufer, J., "Investigation of Turbulent Flow in a Two-Dimensional Channe 1", NACA CR-1053, 1949.

15. Laufer, J., "The Structure of Turbulence in Fully Developed Pipe Flow", NACA TR-1174, 1954.

16. Kim, S.-W., and Chen, C.-P., "A Multiple-Time-Scale Turbulence model Based on Variable Partitioning of the Turbulent Kinetic Energy

Spectrum", NASA CR-179222, In print, 1987, See also AIAA-88-0221, AIAA Aerospace Sciences Meeting, Reno, Nevada, 1988.

17. Irwin, H. P. A. H., "Measurements in a Self-Preserving Plane Wall Jet in a Positive Pressure Gradient", J. Fluid Mech., Vol. 61, pp. 33-63, 1973.

18. Tsiolakis, E. P., Krause, E., and Muller, U. R., "Turbulent Boundary Layer-Wake Interaction" in Bradbury, L. J. S., Durst, F., Launder, B. E., Schmidt, F. W., and Whitelaw, J. H., Eds., Turbulent Shear Flows, Vo1. 4, Springer-Verlag, New York, 1983.

19. Kim, S.-W., and Chen, C.-P., "A Review on Various Flow-Solid Interaction Analysis Methods with Emphasis on Recent Advances in Turbulence Models and Flow Analysis Methods," AIAA-88-3685, First National Fluid Dynamics Conference, Cincinnati, Ohio, 1988.

20. Roback, R. and Johnson B. V., "Mass and Momentum Turbulent Transport Experiments with Confined Swirling Coaxial Jets", NASA CR-168252, 1983.

21. Hanjelic, K., Launder, B. E., and Schiestel, R., "Multiple-Time-Scale Concepts in Turbulent Shear Flows" in Bradbury, L. J. S., Durst, F., Launder, B. E., Schmidt, F. W., and Whitelaw, J. H., Eds., Turbulent Shear Flows, Vo1. 2, 36-49, Springer-Verlag, New York, 1980.

22. Schiestel, R., "Multiple-Time-Scale Modeling of Turbulent Flows in One point Closure", Physics of Fluids, Vol. 30, pp. 722-731, 1987.

23. Schiestel, R., "Multiple-Scale Concept in Turbulence Modelling, II. Reynolds Stresses and Turbulent Fluxes of a Passive Scalar, Algebraic Modelling and Simplified Model using Boussinesq Hypothesis", Journal de Mechanique theorique et appliquee, Vol. 2, No. 4, pp. 601-628, 1983.

24. Wilcox, D. C., "More Advanced Applications of the Multiscale Turbulence Model for Turbulent Flows," AIAA-88-0220, 26th Aerospace Sciences Meeting, Reno, Nevada, 1988.

25. Gibson, M. M., Spalding, D. B., and Zinser, W., "Boundary Layer Calculations using the Hassid-Poreh One-Equation Energy Model," Letters in Heat and Mass Transfer, Vol. 5, pp. 73-80, 1978.

26. Hassid, S., and Poreh, M., "A Turbulent Energy Model for Flows with Drag Reduction," J. Fluid Engineering, Transactions of ASME, pp. 234-241, 1975 . 
27. Acharya, M., and Reynolds, W. C., "Measurements and Predictions of a Fully Developed Turbulent Channel Flow with Imposed Controlled Oscillations," Thermoscience division, Stanford University, Tech. Report TF-8, 1975.

28. Roshko, A., "Structure of Turbulent Shear Flows: A New Look, " J. AIAA, Vo1. 14, No. 10, pp. 1349-1357, 1976.

29. Lumley, J. L., "Turbulence Modelling," J. Applied Mechanics, Transactions of ASME, Vo1. 50, pp. 1097-1103, 1983.

30. Coles, D., "A Model for Flow in Viscous Sublayer," Proceedings of the Workshop on Coherent Structure of Turbulent Boundary Layers, Lehigh University, Bethlehem, Pa., 1978.

31. Kim, S.-W., and Payne, F. R., "Finite Element Analysis of Incompressible Laminar Boundary Layer Flows", Int. J. Nume. Meth. Fluids, 5, 545-560, (1985).

32. Kim, S.-W., and Chen, Y.-S., "A Finite Element Computation of Turbulent Boundary Layer Flows with an Algebraic Stress Turbulence Model", Comput. Meth. Appl. Mech. Engrg., Vol. 66, No. 1, pp. 45-63, 1988.

33. Kline, S. J., Cantwell, B. J., and Lilley, G. M., Eds., The 1980-1981 AFOSR-HTTM Stanford Conference on Complex Turbulent Flows, Vol. I and III, Stanford, California, 1981. 


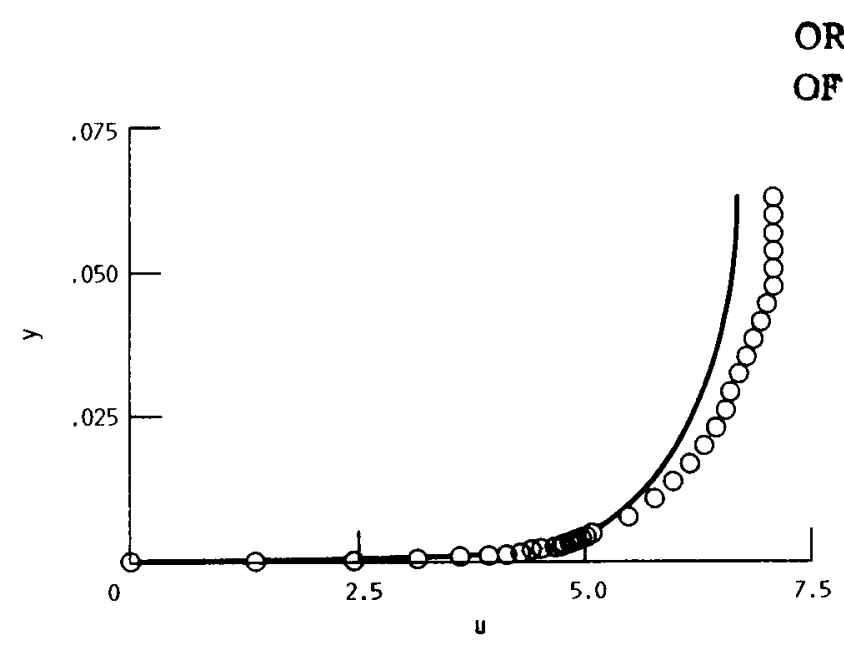

(A) VELOCITY.

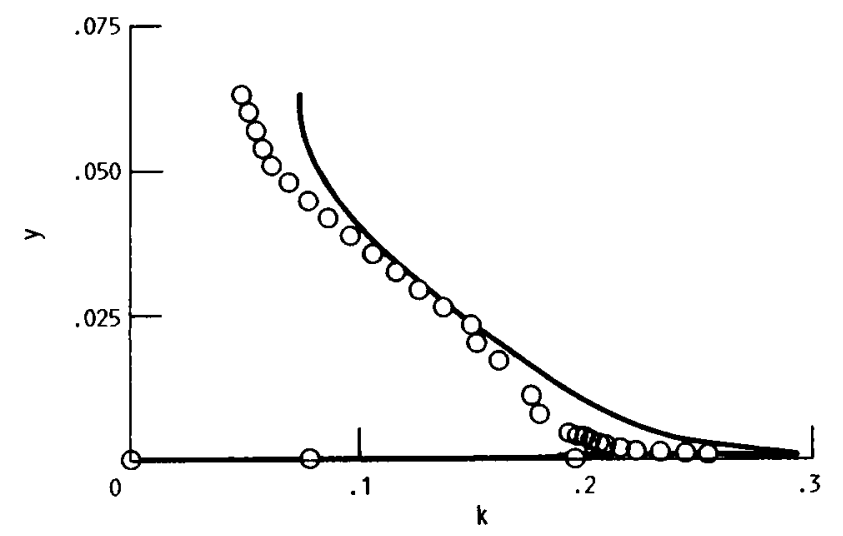

(B) TURBULENT KINETIC ENERGY.

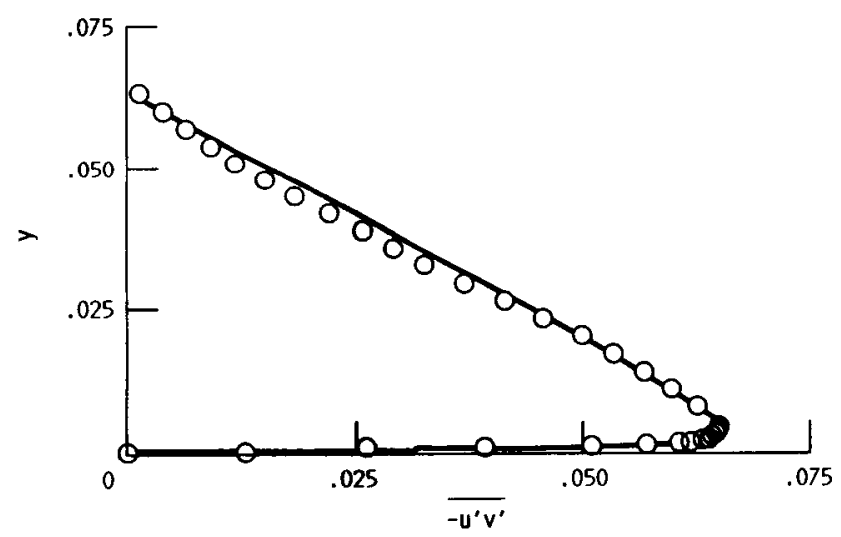

(C) REYMOLDS STRESS.

FIGURE 1. - FULLY DEVELOPED CHANNEL FLOW, O: EXPER IMENTAL DATA (REF. 10), : COMPUTATIONAL RESULT.

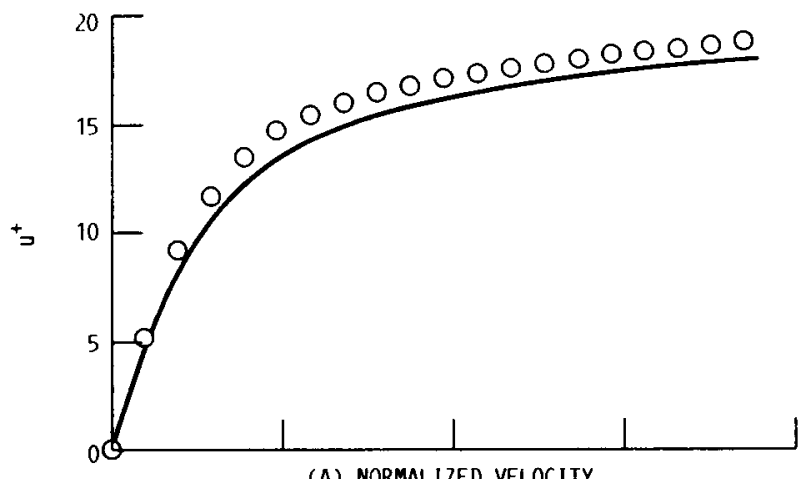

(A) NORMAL IZED VELOCITY.

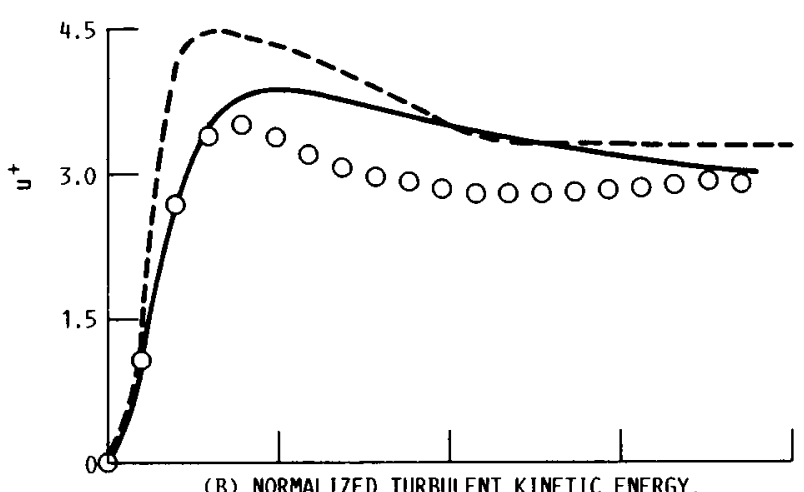

(B) NORMAL IZED TURBULLENT KINETIC ENERGY.

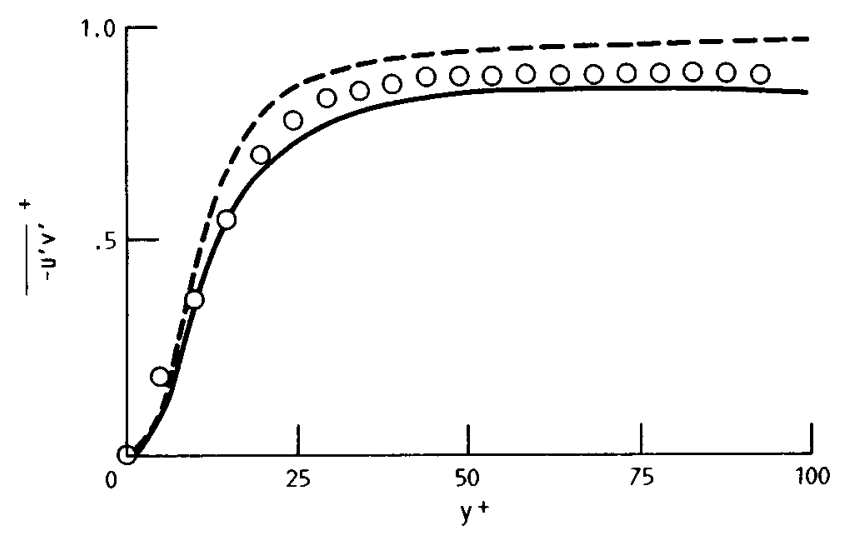

(C) NORMAL IZED REYNOLDS STRESS.

FIGURE 2. - FULLY DEVELOPED CHANNEL FLOW, O: EXPERIMENTAL DATA (REF, 10), - COMPUTATIONAL RESULT,

--- : SEMI-EMPERICAL DATA (REF. 7). 
ORIGINAL PAGE IS

OF POOR QUALITY
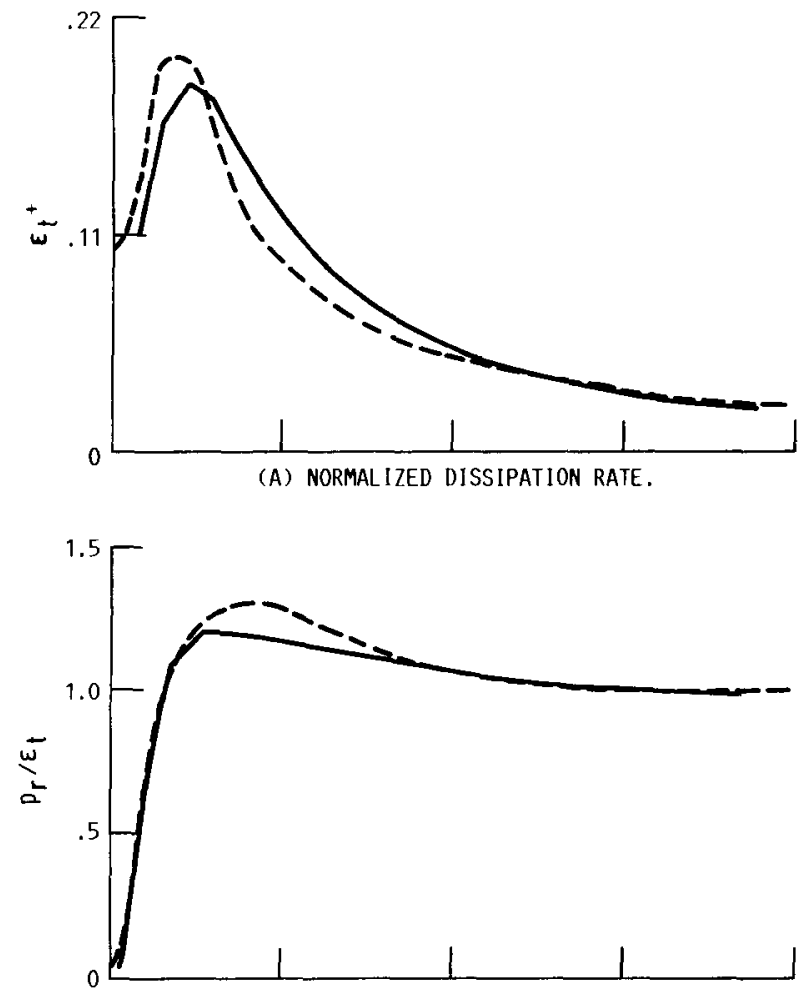

(B) Ratio OF PRODUCTION RATE tO dissipation Rate.

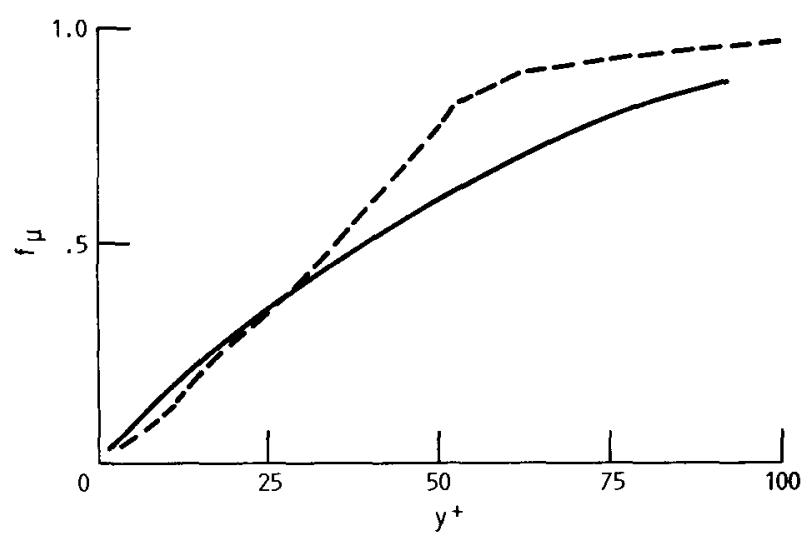

(C) WALL DAMPING FUNCTION.

FIGURE 3. - FULLY DEVELOPED CHANNEL FLOW. - : COMPUIATIONAL RESULT, - - : SEMI-EMPERICAL DATA (REF. 7).

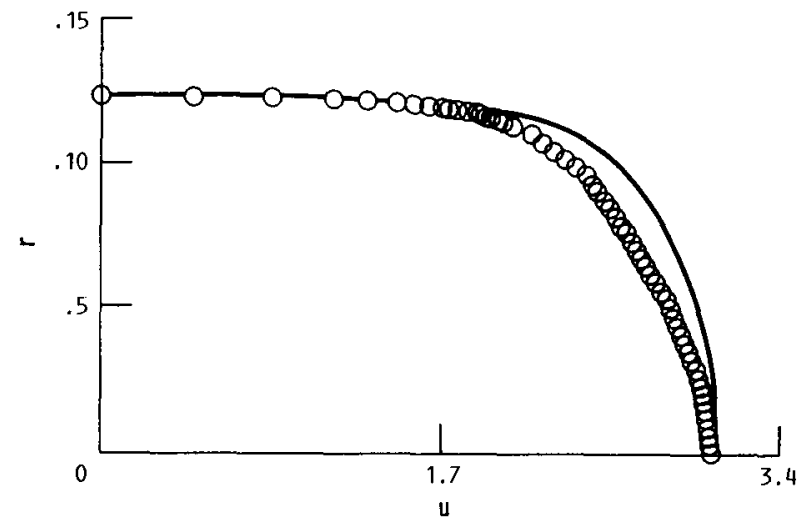

(A) VELOCITY.

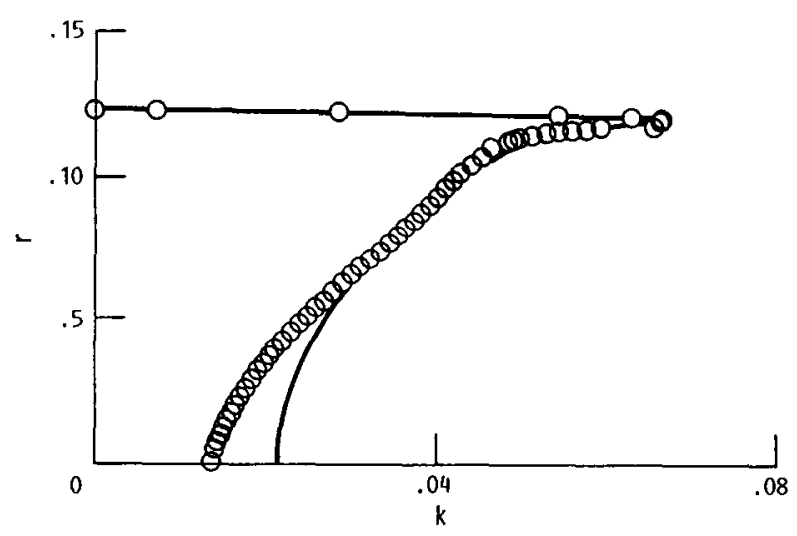

(B) TURBULENT KINETIC ENERGY.

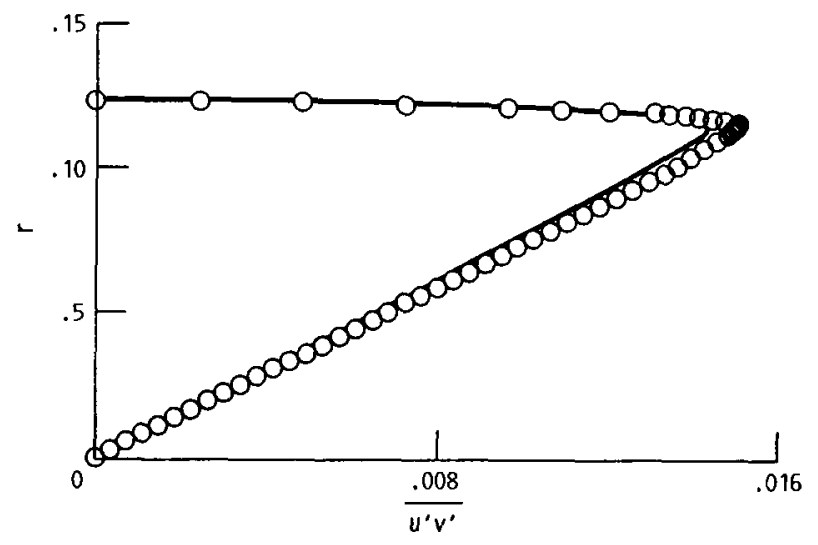

(C) REYNOLDS STRESS.

FIGURE 4. - FULLY DEVELOPED PIPE FLOW FOR RE $=50000$. O: EXPERIMENTAL DATA (REF. 11), - COMPUTATIONAL RESULT. 


\section{ORIGINAL PAGE IS}

OF POOR QUALITY

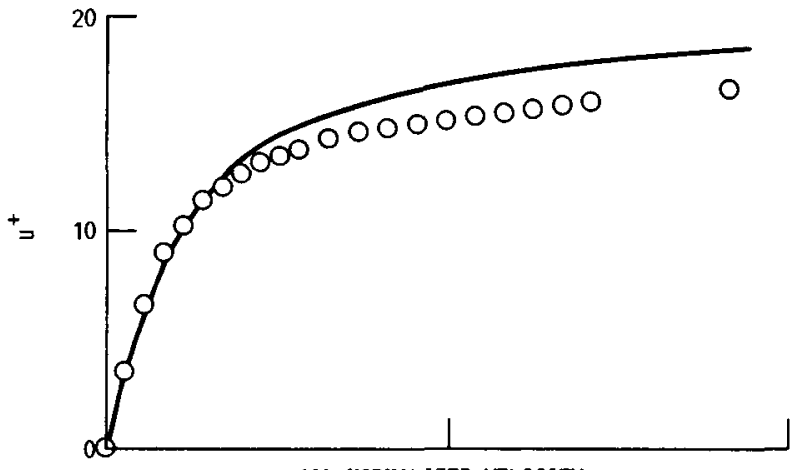

(A) NORMAL IZED VELOCITY.

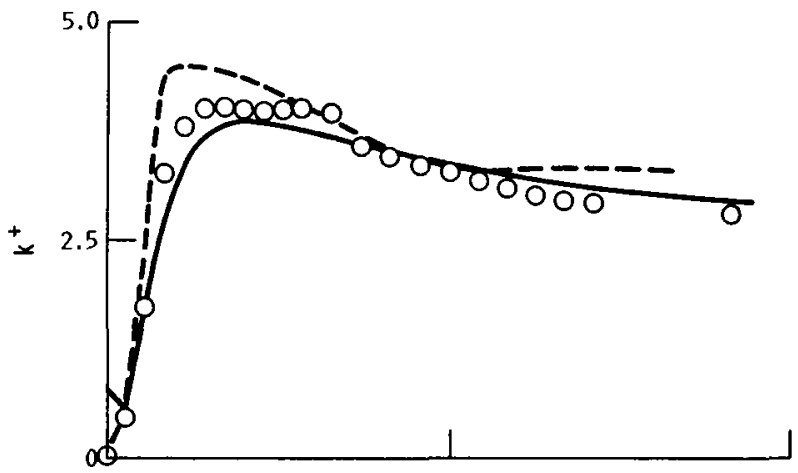

(B) NORMALIZED TURBULENT KIMETIC ENERGY.

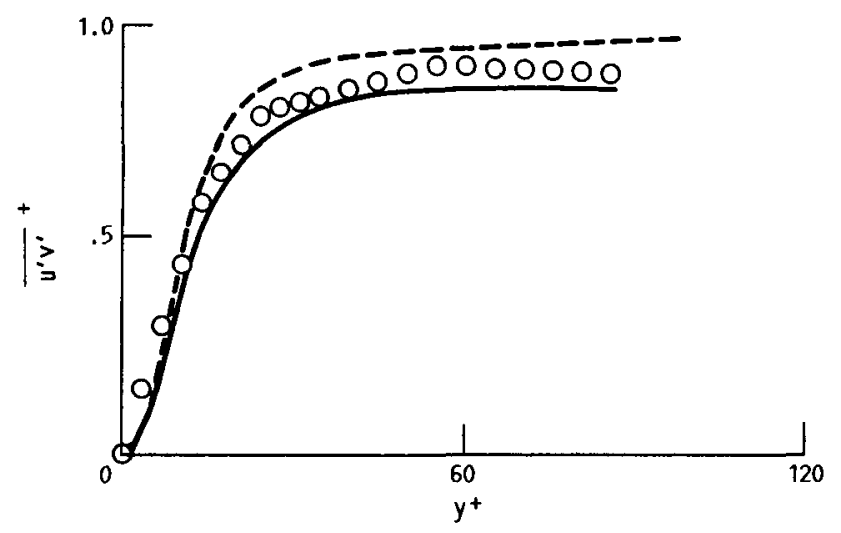

(C) NORMALIZED REYNOLDS STRESS.

FIGURE 5. - FULLY DEVELOPED PIPE FLOR FOR RE $=50000$. $O$ : EXPERIMENTAL DATA (REF. 11), — : COMPUTATIONAL RESULT, - : : SEMI-EMPERICAL DATA (REF, 7),

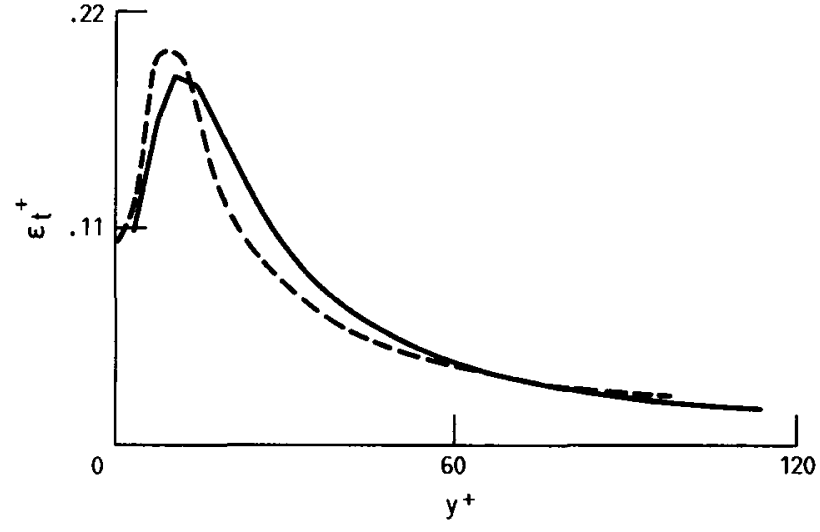

(A) NORMALIZED DISSIPATION RATE.

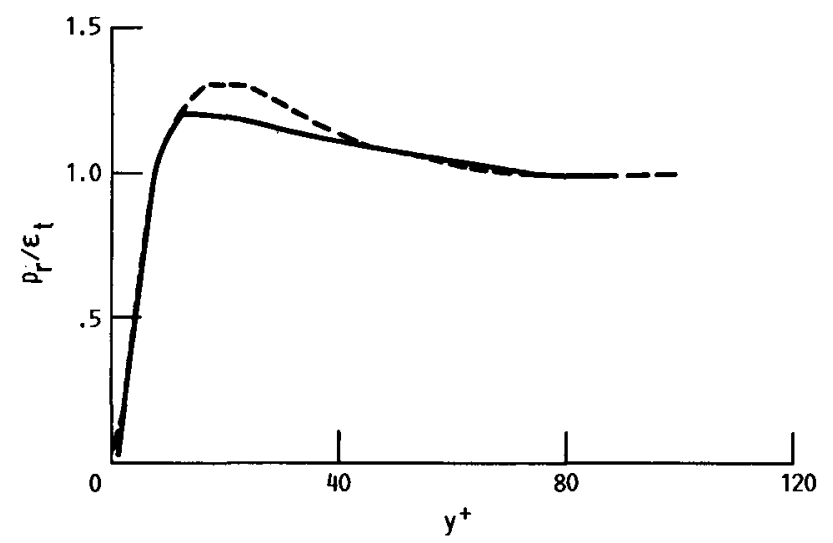

(B) RATIO OF PRODUCIION RATE TO DISSIPATION RATE.

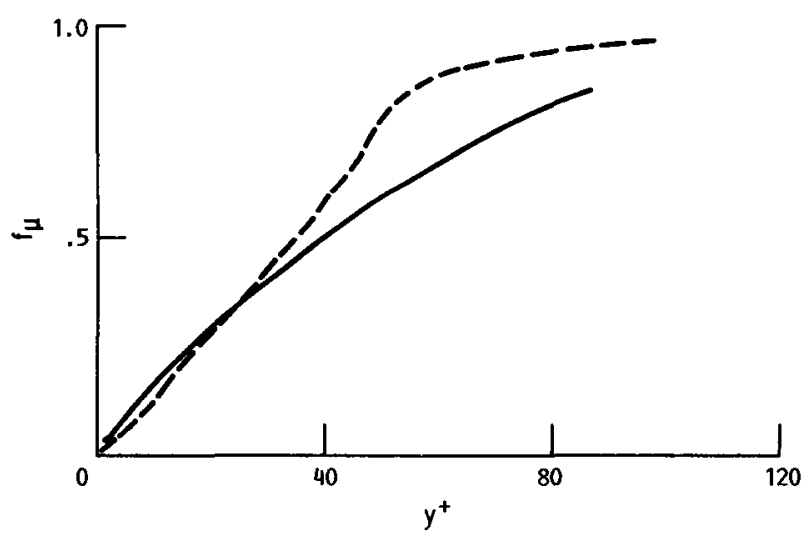

(C) HALL DAMPING FUNCTION.

FIGURE 6. - FULLY DEVELOPED PIPE FLON FOR RE $=50000$. : COMPUTATIONAL RESULT, - - : SEMI-EMPERICAL DATA (REF. 7 ). 


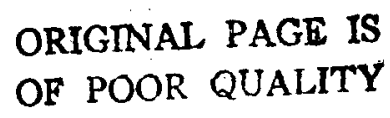

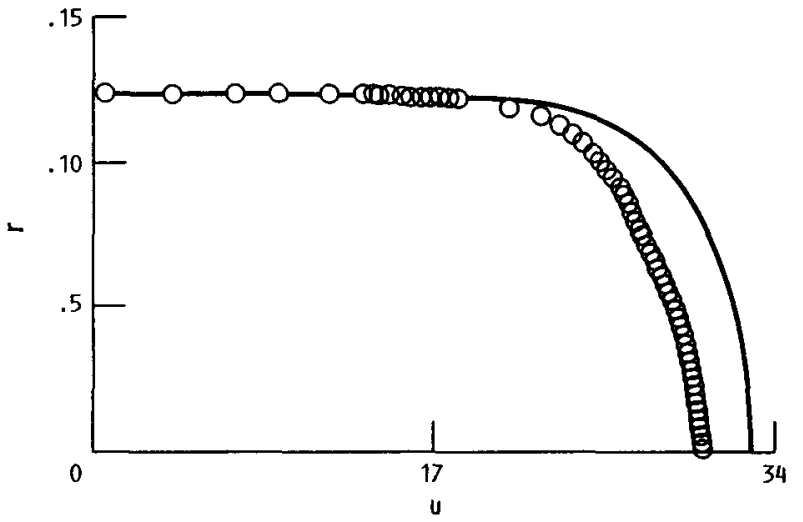

(A) VELOCITY.

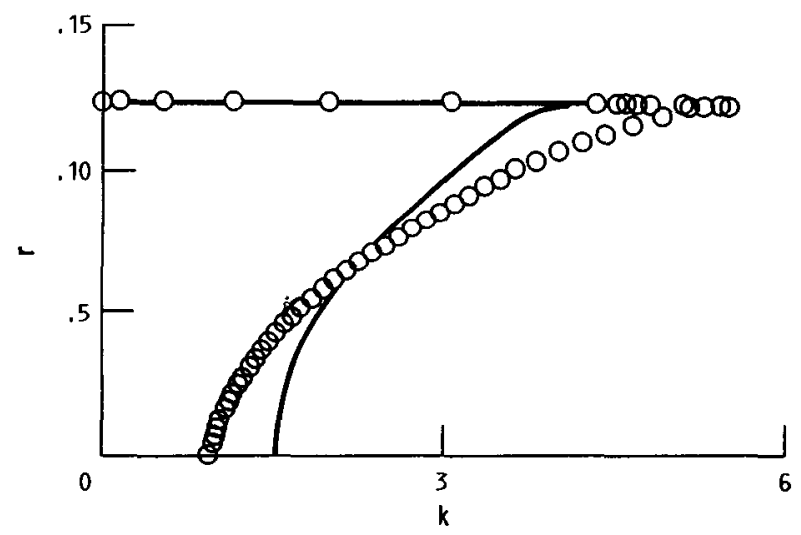

(B) TURBULENT KINETIC ENERGY.

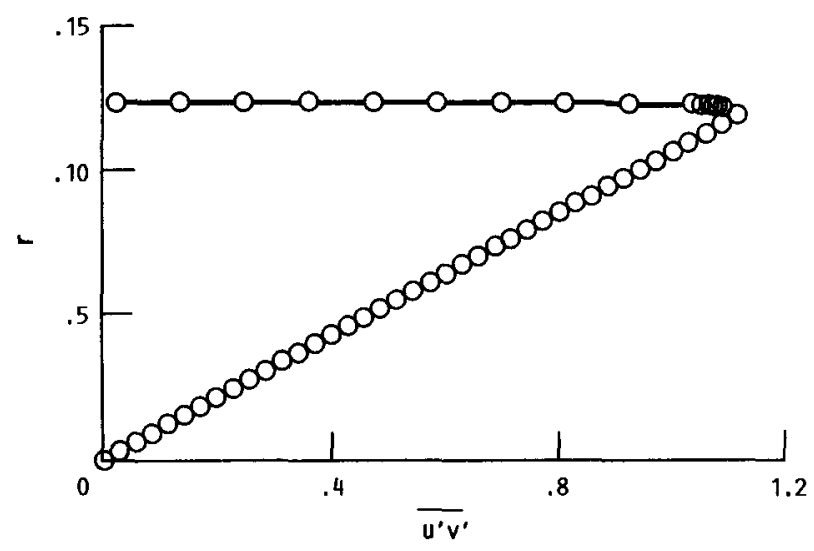

(C) REYNOLDS STRESS.

FIGURE 7. - FULLY DEVELOPE PIPE FLOW FOR RE $=500000$. O: EXPERIMENTAL DATA (REF. 11), - COMPUTATIONAL RESULT.
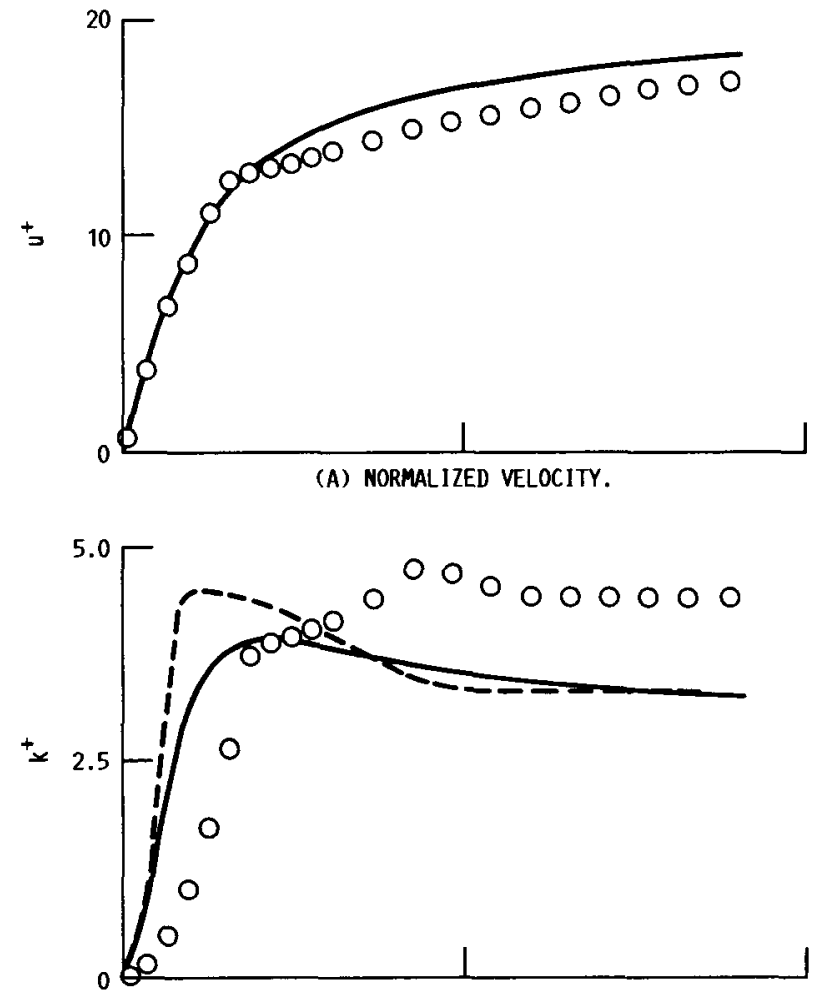

(B) NORMALIZED TURBULENT KINETIC ENERGY.

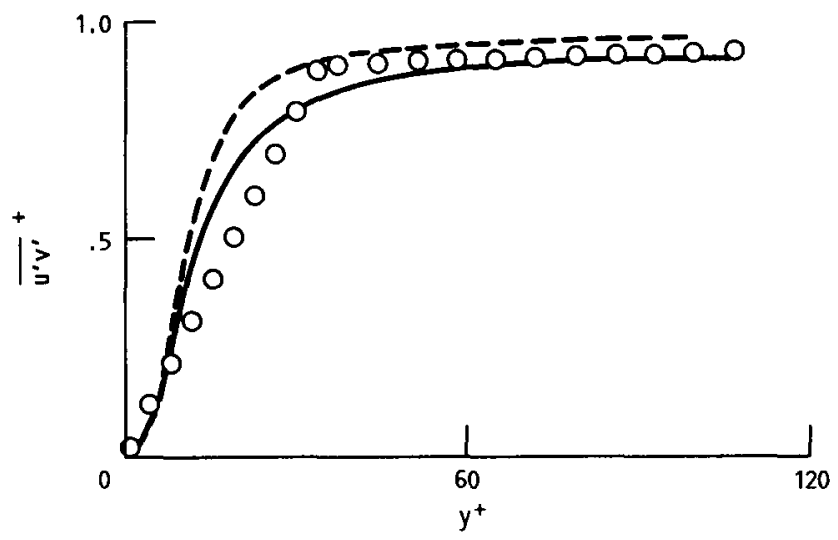

(C) NORMAL IZED REYNOLDS STRESS.

FIGURE 8. - FULLY DEVELOPED PIPE FLOW FOR RE $=500000$, O: EXPERIMENTAL DATA (REF.) 11, - COMPUTATIONAL RESULT, --- : SEMI-EMPERICAL DATA (REF. 7). 
ORIGINAL PAGE IS

OF POOR QUALITY

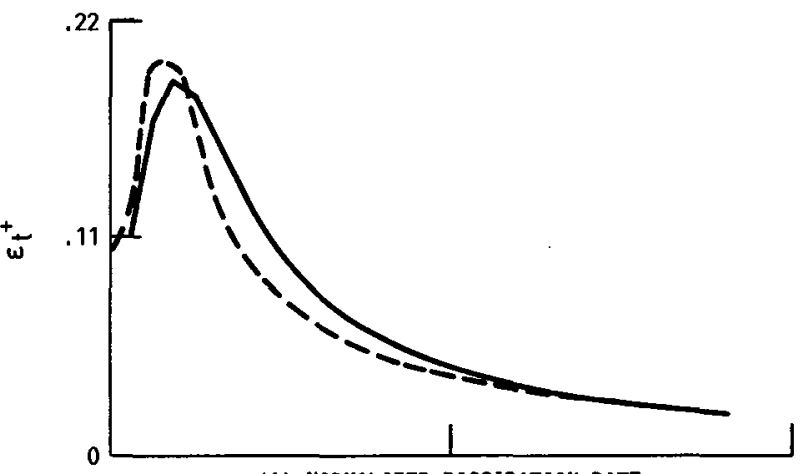

(A) NORMALIZED DISSIPATION RATE.

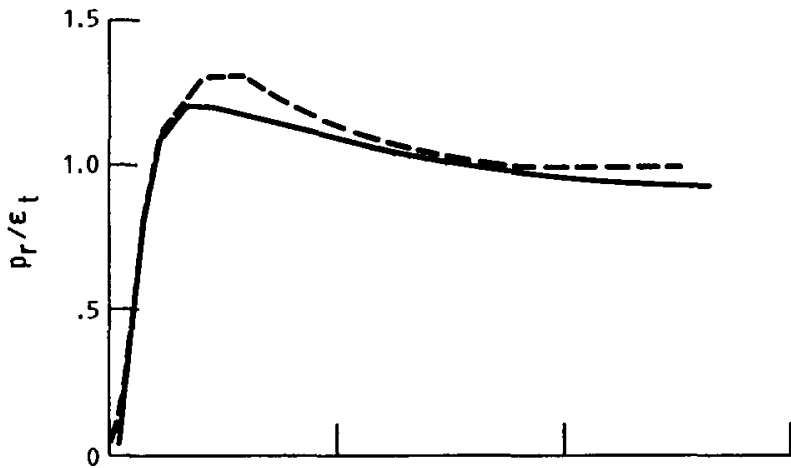

(B) RATIO OF PRODUCTION RATE TO DISSIPATION RATE.

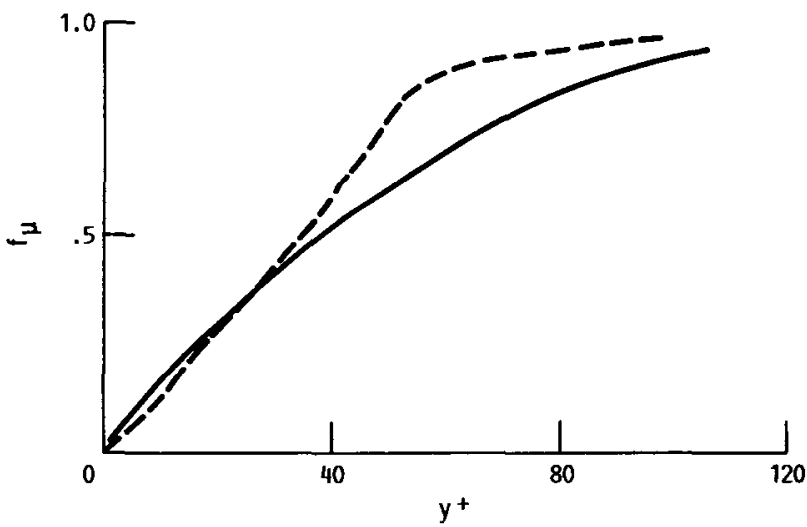

(C) WALL DAMPING FUMCTION.

FIGURE 9. - FULLY DEVELOPED PIPE FLON FOR RE $=500000$. - COMPUTATIONAL RESULT, - - : SEMI-EMPERICAL DATA (REF, 7), 


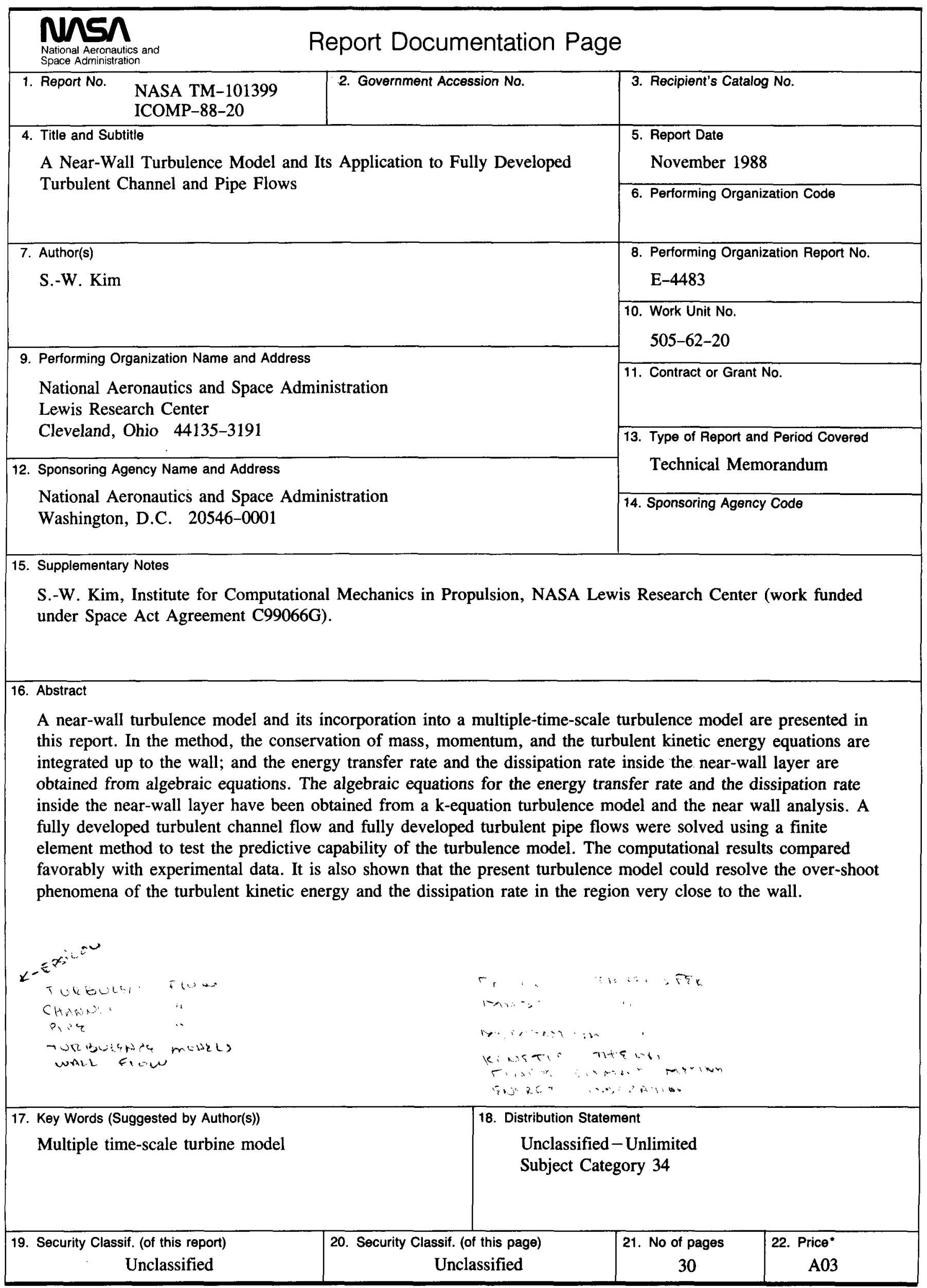

